\title{
Non-standard Aspects of Fibonacci Type Sequences
}

\author{
Dedicated to Hellmuth Stachel on the occasion of his 75th birthday
}

\begin{abstract}
Non-standard Aspects of Fibonacci Type Sequences

\section{ABSTRACT}

Fibonacci sequence and the limit of the quotient of adjacent Fibonacci numbers, namely the Golden Mean, belong to general knowledge of almost anybody, not only of mathematicians and geometers. There were several attempts to generalize these fundamental concepts which also found applications in art and architecture, as e.g. number series and quadratic equations leading to the so-called "Metallic means" by V. DE SPINADEL [8] or the cubic "plastic number" by VAN DER LAAN [5] resp. the "cubi ratio" by L. ROSENBUSCH [7]. The mentioned generalisations consider series of integers or real numbers. "Non-standard aspects" now mean generalisations with respect to a given number field or ring as well as visualisations of the resulting geometric objects. Another aspect concerns Fibonacci type resp. Padovan type combinations of given start objects. Here it turns out that the concept "Golden Mean" or "van der Laan Mean" also makes sense for vectors, matrices and mappings.
\end{abstract}

Key words: generalized Fibonacci sequence, Golden Mean, non-Euclidean geometry, number field, ring

MSC2010: 51Mxx

\section{Introduction}

There exists already a huge set of references concerning the Fibonacci sequence and the Golden Mean and some generalisations of these concepts, most of them only repeating results of former works, such that it seems to be hopeless to add something essentially new to that topic. We shall start with basic facts about the Fibonacci sequence and the limit of adjacent Fibonacci numbers, namely the Golden Mean. Subsequently, we shall have a short look at the main traces of existing generalisations of these basic properties and concepts before adding another generalisation type. Finally, it will turn out that these pro-

\begin{abstract}
Nestandardni pristupi nizovima Fibonaccijevog tipa

SAŽETAK

Fibonaccijev niz i zlatni rez, limes kvocijenata susjednih Fibonaccijevih brojeva, su pojmovi poznati ne samo matematičarima i geometričarima, već gotovo svima. Oni svoju primjenu nalaze u umjetnosti i arhitekturi. Poznato je nekoliko pokušaja poopćenja ovih pojmova, kao što su nizovi brojeva i kvadratne jednadžbe koje rezultiraju takozvanim "metalnim rezovima" V. DE SPINADEL [8], ili kubni "plastični broj" VAN DER LAANA [5], odnosno "kubni omjer" L. Rosenbuscha [7]. Spomenuta se poopćenja odnose na nizove cijelih ili realnih brojeva. "Nestandardnim pristupima" ovdje smatramo poopćenja u odnosu na dano polje ili prsten brojeva, kao i na vizualizaciju dobivenih geometrijskih objekata. Idući se pristup odnosi na Fibonaccijev, odnosno Padovanov tip kombinacija danih početnih objekata. Pokazuje se da pojam zlatnog reza ili van der Laanovog reza ima smisla promatrati i za vektore, matrice i preslikavanja.
\end{abstract}

Ključne riječi: popćeni Fibonaccijev niz, zlatni rez, neeuklidska geometrija, polje brojeva, prsten

posed additional points of view are very natural and they are unifying consequences of what is already known.

\section{Basic facts about Fibonacci sequences and the Golden Mean}

The Fibonacci sequence is based on the recursive definition

$F_{n+1}=F_{n}+F_{n-1},(n \in \mathbb{N}), \quad F_{1}=1, \quad F_{0}=0$.

Therewith, one receives the standard representation $\{\mathbf{0}, \mathbf{1}, 1,2,3,5,8,13,21,34,55,89, \ldots\}$. 
The limit of adjacent Fibonacci numbers for $n \rightarrow \infty$ is, therefore,

$\phi:=\lim _{n \rightarrow \infty} \frac{F_{n+1}}{F_{n}}=\lim _{n \rightarrow \infty} \frac{F_{n}+F_{n-1}}{F_{n}}=1+\lim _{n \rightarrow \infty} \frac{F_{n-1}}{F_{n}}=1+\frac{1}{\phi}$,

and $\phi$ solves the quadratic equation

$x^{2}-x-1=0$.

Remark 1 This limit is independent of the two start elements, i.e. 0 and 1 can be replaced by any numbers $a_{0}, a_{1} \in \mathbb{R}$ (not both 0$)$. By recursion we receive $a_{n}=$ $F_{n} a_{1}+F_{n-1} a_{0}$, and obviously we get

$\lim _{n \rightarrow \infty} \frac{a_{n}}{a_{n-1}}=\lim _{n \rightarrow \infty} \frac{\left(\frac{F_{n}}{F_{n-1}} a_{1}+a_{0}\right) F_{n-1}}{\left(\frac{F_{n-1}}{F_{n-2}} a_{1}+a_{0}\right) F_{n-2}}=\frac{\phi a_{1}+a_{0}}{\phi a_{1}+a_{0}} \phi=\phi$.

In the following we will interpret $a_{n+1}$ as a special linear combination, say a "Fibonacci combination", of the pair of start elements $a_{0}, a_{1}$. To present an example we remind to the "Lucas numbers", which use the start elements 2,1 (see e.g. [4]).

Remark 2 The Golden Mean value $\phi$ is the well-known result of the periodic continuous fraction

$\phi=1+\frac{1}{1+\frac{1}{1+\frac{1}{1+\cdot}}}=1,618 \ldots$

Remark 3 The classical Greek point of view intersects a given segment $[A, B]$ (of length 1 ) into two segments $M$ (the maior) and $m$ (the minor) such that

$m: M=M: 1=1-M: M$

Therewith, it follows the quadratic condition

$M^{2}+M-1=0$,

which has the positive solution $M=0,618 \cdots=\frac{1}{\phi}$. This value is also considered as the "Golden Mean" and it suits to the line of numbers with $A:=0, B:=1, M:=0,618 \ldots$, while the affine ratio $R(M, A, B)$ is the negative of this value.

Remark 4 It is possible to directly calculate the $n^{\text {th }} \mathrm{Fi}$ bonacci number $F_{n}$ as

$F_{n}=\frac{(1+\sqrt{5})^{n}-(1-\sqrt{5})^{-n}}{2^{n} \sqrt{5}}$.

There is an obvious connection of $F_{n}$ to the Pascal triangle, (Fig. 1 and [15]) that leads to an expression for $F_{n}$ also as a sum of binomial coefficients as

$F_{n}=\sum_{p+q=n-2}\left(\begin{array}{l}p \\ q\end{array}\right)$.

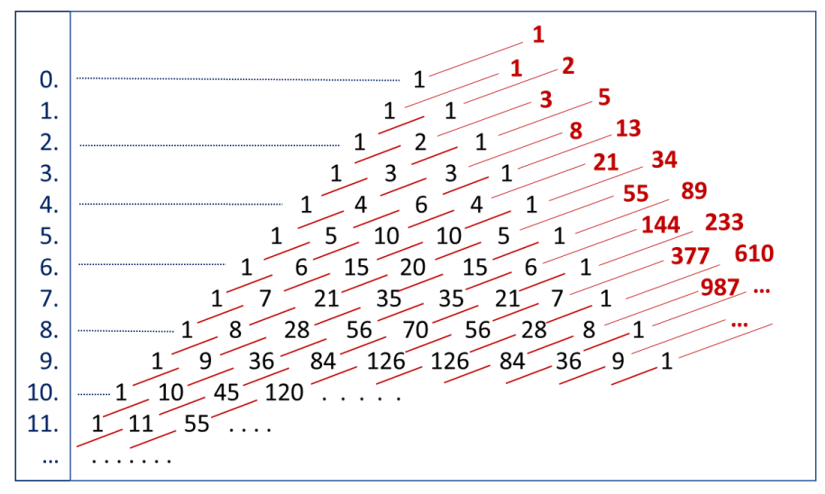

Figure 1: The Fibonacci numbers $F_{n}$ can be received as transversal sums of binomial coefficients as indicated in the Pascal triangle.

Remark 5 The Fibonacci sequence $\{\mathbf{0}, \mathbf{1}, 1,2,3,5,8, \ldots\}$ can be interpreted as an approximation of the geometric sequence $\left\{1, \phi, \phi^{2}, \phi^{3}, \phi^{4}, \ldots\right\}$. By the way, Lucas numbers are an even better approximation of this geometric sequence (c.f. [15]).

We conclude this chapter noticing that the Golden Mean can be connected

(i) with the Fibonacci sequence,

(ii) with the quadratic equation (3), and

(iii) with the periodic continued fraction (4).

Generalisations of the Golden Mean are based on each of these mentioned connections.

\section{Some known generalisations of the Fi- bonacci sequence and the Golden Mean}

a) Generalisations of the Fibonacci sequence

As an example we mention the Padovan sequence, which leads to van der Laan's plastic number as a 3Dgeneralisation of the Golden Mean:

By replacing (1) by the rule

$P_{n+1}=P_{n-1}+P_{n-2}$

and using the start elements $\mathbf{0}, \mathbf{1}, \mathbf{1}$ we get the "Padovan standard sequence" $\{\mathbf{0}, \mathbf{1}, \mathbf{1}, 1,2,2,3,4,5,7,9,12,16,21$, $28,37, \ldots\}$. The limit of the ratio of consecutive Padovan numbers $P_{n+1}: P_{n}$ is the (positive) solution $\psi$ of the cubic equation

$x^{3}-x-1=0$.

This solution $\psi=1,324717958 \ldots$ is called "van der Laan's plastic number", as he used it as a 3D-replacement for the Golden Mean in his architectural design (see [5], [6], [10], and [16]). 
Remark 6 Using arbitrary start values $a_{0}, a_{1}, a_{2}$ (not all $\left.a_{i}=0\right)$ we get the recursion formula

$a_{n+1}=P_{n-3} a_{0}+P_{n-1} a_{1}+P_{n-2} a_{2}$,

and again the limit of the quotient of consecutive numbers $a_{n+1}, a_{n}$ is van der Laan's plastic number $\psi$. As an example one could mention the "Perrin sequence", which uses the start elements $\mathbf{3 , 0 , 2}$ instead of $\mathbf{0 , 1}, \mathbf{1}$.

Remark 7 The Padovan sequence can also be connected to the Pascal triangle by choosing a special "skew" direction for summing up binomial coefficients, similar to the Fibonacci case Fig. 1, c.f. [16]. This gives rise to a procedure to construct other maybe interesting sequences derived from the Pascal triangle.

Remark 8 To calculate the polynomial (7) one can use the linear mapping, which maps the vector $\left(P_{n}, P_{n-1}, P_{n-2}\right)^{\top}$ to the vector $\left(P_{n+1}, P_{n}, P_{n-1}\right)^{\top}$ :

$\left(\begin{array}{c}P_{n+1} \\ P_{n} \\ P_{n-1}\end{array}\right)=\left(\begin{array}{lll}0 & 1 & 1 \\ 1 & 0 & 0 \\ 0 & 1 & 0\end{array}\right)\left(\begin{array}{c}P_{n} \\ P_{n-1} \\ P_{n-2}\end{array}\right) \Longrightarrow$

char.Pol. : $\left|\begin{array}{ccc}-x & 1 & 1 \\ 1 & -x & 0 \\ 0 & 1 & -x\end{array}\right|=0=-x^{3}+x+1$.

\section{b) Generalisations of the equation defining the Golden} Mean

V. de Spinadel's Metallic Means

A simple way to define generalisations of the Golden Mean deals with (positive) solutions of quadratic equations by allowing arbitrary (positive or negative) integer coefficients for the characteristic equation

$x^{2}-p x-1=0, \quad$ or $\quad x^{2}-x-q=0, \quad(p, q \in \mathbb{Z})$,

c.f. [8] and [9]. For the solutions of (10) with $p=$ $2,3,4, \ldots$ V. Spinadel coined the concepts "Silver Mean" $\sigma_{2}$, "Bronze Mean" $\sigma_{3}$, "Copper Mean" $\sigma_{4}$ resp. "Metallic Mean" $\sigma_{p}$ for general $p \in \mathbb{N}$. The Silver Mean $\sigma_{2}:=$ $1+\sqrt{2}$ occurs as the affine ratio of the $2^{\text {nd }}$ diagonal of a regular octagon to its side and is, therefore, omnipresent in medieval architecture.

The Metallic Means take the values of periodic continued fractions of type

$\sigma_{p}=p+\frac{1}{p+\frac{1}{p+\frac{1}{p+}}}, \quad p \in \mathbb{N}$.

Obviously, problems in physics, mathematics, and geometry, which result in a quadratic equation with rational or especially integer coefficients can be interpreted as applications of what can be called the "Generalised Metallic Means Family", c.f. [14], where the authors generalise a result of G. Odom [3] connecting the Golden Mean to an equilateral triangle and its circumcircle, see Fig. 2, to regular polyhedra and their circum-sphere.
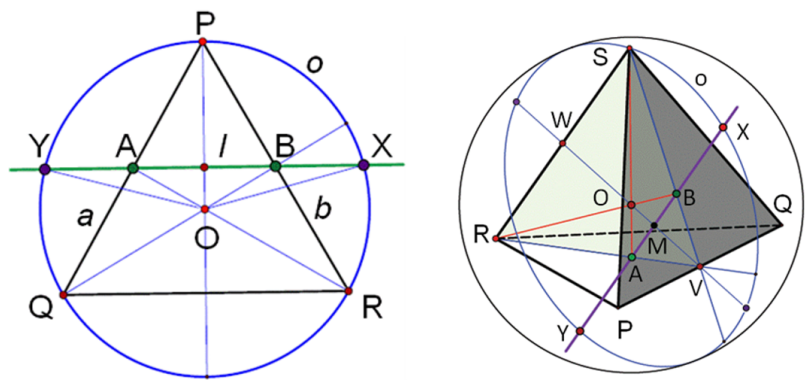

Figure 2: Odom's discovery of the Golden Mean (left) and one of many possible generalisations (right).

Generalising the equation (3) to irreducible equations of higher degree

H. van der Laan seems to be the first following this idea with using the cubic equation (7) for defining what he called the "plastic number" $\psi=1,324717958 \ldots$. But independent of him L. Rosenbusch [7] (see also [10]), too, looked for a 3D-analogue of the Golden Mean which he called "cubi ratio" $\rho:=0,6823278040 \ldots$, and which is the (positive) solution of the cubic equation

$x^{3}+x-1=0$.

Putting $y:=\frac{1}{x}$ in (7) and (7') we get the equations $y^{3} \pm$ $y^{2}-1=0$, and a somehow natural generalisation of the equations (7) and (7') would be

$x^{d} \pm x-1=0 \quad$ or $\quad x^{d} \pm x^{d-1} \pm \cdots \pm x-1=0$.

As many of the classical geometric problems from the ancient Greeks lead to cubic equations, one could consider general cubic equations, too, and construct the Fibonacci type sequence to it. This means to reconstruct the linear mapping (9) to a given characteristic polynomial

$x^{3}-p x^{2}-q x-r=0, \quad(p, q, r \in \mathbb{R})$,

with real solution $x_{0}$. As (12) is of degree $d=3$, we put $S_{n+1}=a S_{n-2}+b S_{n-1}+c S_{n}$. Division by $S_{n-2}$ and calculating the limits for $n \rightarrow \infty$ gives $x_{0}^{3}=a+b x_{0}+c x_{0}^{2}$, and we find $a:=r, b:=q, c:=p$. Note that a Fibonacci type sequence should have at least two non-zero values $a, b, c$. These considerations can be formulated as

Result 1: Given an (irreducible) polynomial $P[x]$ of degree $d$, then it is possible to construct a Fibonnacci type sequence $\left\{\ldots, P_{n-d}, P_{n-d+1}, \ldots, P_{n-1}, P_{n}, \ldots\right\}$ to it, such that 
$P[x]=0$ is the characteristic polynomial of the linear mapping

$\left\{P_{n-d}, P_{n-d+1}, \ldots, P_{n-1}, P_{n}\right\} \mapsto\left\{P_{n-d+1}, \ldots, P_{n-1}, P_{n}, P_{n+1}\right\}$.

If the sequence $P_{n+1} / P_{n}$ is convergent, then the limit $\lim _{n \rightarrow \infty} P_{n+1} / P_{n}$ is a (real) solution of $P[x]=0$. Therewith, we get an additional method to find Zeros of a polynomial, besides the well-known and more general methods "regula falsi" and "Newtons method".

Remark 9 By a Tschirnhaus-Bring-Jerrard transform [1] it is always possible to transform an equation $x^{d}+$ $p_{1} x^{d-1}+p_{2} x^{d-2}+\cdots+p_{d-1} x+p_{d}=0$ into one with $p_{1}=p_{2}=0$. For a cubic equation this would result in $x^{3}=q$, which is not a proper form for constructing a Fibonacci type sequence.

As an example, the equation $x^{3}=2$, describing the classical problem of doubling the cube, can be solved via the cubic equation $y^{3}-y^{2}+y-\frac{1}{3}=0$ with $y=\frac{1-x}{x}$.

Another example is the trisection of an angle $\alpha$ : Putting $x=\tan \alpha, a=\tan 3 \alpha$ the corresponding cubic equation becomes $x^{3}-3 a x^{2}-3 x+a=0$.

The Cubus Simus, the "snub cube", resp. the regular heptagon are connected to the cubic equations $x^{3}+x^{2}+$ $3 x-1=0$ resp. $x^{3}+x^{2}-2 x-1=0$, while $x^{3}-x^{2}+$ $2 x-1=0$ describes the axis-ratio of quarter-ellipses of $a C^{2}$-continuous bi-arc spiral consisting of such quarter ellipses. The latter equation can be transformed into (7) by the Tschirnhaus-Bring-Jerrard transformation and thus relates to van der Laan's Plastic Number $\psi$ (c.f. [14]).

\section{c) Continued fractions and their iterations}

A theorem by Euler-Lagrange states that periodic continued fractions only lead to quadratic equations. This means that such fractions only can take values of the Generalized Metallic Means Family. From the decimal representation of, e.g., van der Laan's Plastic Number $\psi$, one could calculate finite approximations of the surely non periodic continued fraction of $\psi$. But one could generalise the concept "periodic continuous fraction" using reals instead of natural numbers as coefficients and iterate such a fraction as e.g.

$$
a_{i+1}=a_{i}+\frac{1}{a_{i}+\frac{1}{a_{i}+\frac{1}{a_{i}+} \cdot}}, \quad a_{i} \in \mathbb{R}, \quad a_{0}=1 .
$$

In [12] the limit of this special sequence of periodic continued fractions is considered and turns out to be $\sqrt{2} / 2$. This iteration process can also be seen as a sequence of quadratic equations (10) where the coefficient $p$ is consecutively replaced by the (positive) solution of the former equation.

\section{Fibonacci sequences and finite structures}

\section{a) Elliptic and spherical Geometry}

In [13] visualisations of the Fibonacci sequence and the Golden Mean in non-Euclidean geometries were shown with one exception: the elliptic geometry. The elliptic lenght $L$ of a line is $\pi$, and thus finite!

There seem to be two "natural" possibilities to handle Fibonacci numbers $F_{n}^{e l}$ in elliptic resp. spherical geometry:

(i) $F_{n}^{e l}:=F_{n} \bmod L$.

(ii) $F_{n}^{e l}:=F_{n} \cdot \frac{L}{F_{n+1}}$.

The way (i) seems not to be considered yet, while (ii) can be used to define the well-known "Golden Angle" as

$\alpha:=2 \pi-\lim _{n \rightarrow \infty} 2 F_{n}^{e l}=137,520^{\circ}$.

The usual definition of the Golden Angle devides the circumference of the unit circle into two angle-segments $2 \pi-\alpha$ and $\alpha$ such that $(2 \pi-\alpha): \alpha=\phi$. Obviously (ii) connects this definition to the circular model of the elliptic line. For the first stages $n=1,2,3$, of (ii) one may observe that the ratio of the regular $(n+1)$-gon's side $s_{n+1}$ to its $n$-diagonal $d_{n}$ takes remarkable values $r_{n}=d_{n}: s_{n+1}$, see Fig. 3: For the regular pentagon this ratio is the wellknown Golden Mean, for the regular octagon it is the Silver Mean! As for $n \rightarrow \infty$ this ratio $r_{n} \rightarrow \infty$ it seems more reasonable to calculate the ratios $R_{n}=d_{n}:\left((n-1) \cdot s_{n+1}\right)$ and their limit $\theta$

$$
\begin{aligned}
\theta & :=\lim _{n \rightarrow \infty} R_{n}=\lim _{n \rightarrow \infty} \frac{F_{n-1} \cdot \sin \left(\frac{\pi}{F_{n+1}}\right)}{\pi \cdot \frac{F_{n-1}}{F_{n+1}}}=\frac{\sin \left(\pi / \phi^{2}\right)}{\pi / \phi^{2}} \\
& =0,78185913047 \ldots
\end{aligned}
$$

which is somehow a "golden" number, too.
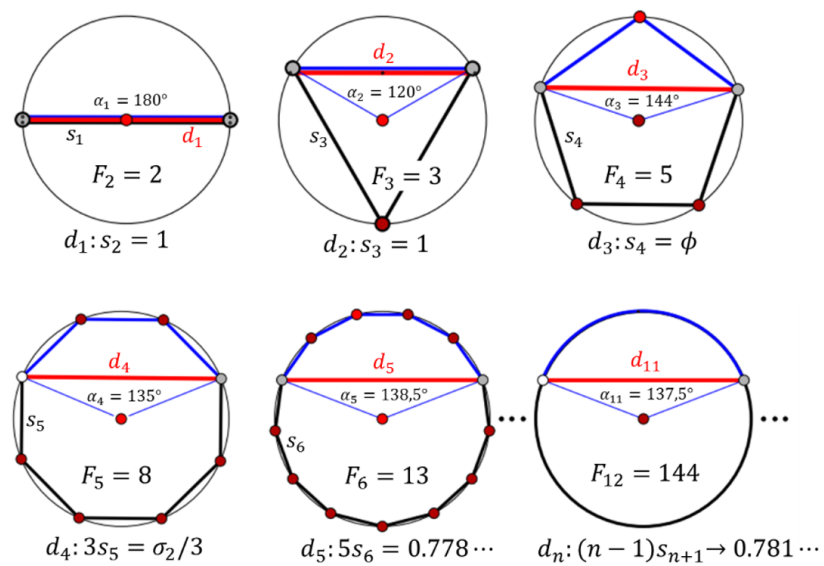

Figure 3: $F_{n+1}$-gons and the ratios of diagonals $d_{n}$ (red) and sides $s_{n+1}$ (blue). 


\section{b) Finite fields and Fibonacci numbers}

We consider the Galois fields $G F(p), p$ prime, $p \in \mathbb{N}$. Here it seems to be natural to use the way (i) of the former subchapter (a) to define Fibonacci numbers $F_{n}^{p}$ :

$F_{n}^{p}:=F_{n} \bmod p=F_{n-1}^{p}+F_{n-2}^{p}$.

Obviously the sequence of Fibonacci numbers $F_{n}^{p}$ is periodic. The behaviour shall be visualised by the following examples:

$$
\begin{aligned}
p=5: & \mathbf{0}, \mathbf{1}, 1,2,3,0,3,3,1,4,0,4,4,3,2,0,2,2,4, \\
& 1, \mathbf{0}, \mathbf{1}, 1, \ldots \\
& \text { Lenght of period is } 20, \text { all numbers of } \\
& G F(5) \text { occur. }
\end{aligned}
$$

$p=7: \mathbf{0}, \mathbf{1}, 1,2,3,5,1,6,0,6,6,5,4,2,6,1, \mathbf{0}, \mathbf{1}, 1, \ldots$ Lenght of period is 16 , all numbers of $G F(7)$ occur.

$p=11: \mathbf{0}, \mathbf{1}, 1,2,3,5,8,2,10,1, \mathbf{0}, \mathbf{1}, 1, \ldots$

Lenght of period is 10 , not all numbers of $G F(11)$ occur! (4, 6, 7, 9 do not occur.)

Other start elements, e.g. 4, 6: 4, 6, 10, 5, 4, 9, 2, $0,2,2, \mathbf{4}, \mathbf{6}, \ldots$

Again the length of period is 10 . There are four numbers of $G F(11)$ that do not occur: $1,3,7,8$.

$p=13: \mathbf{0}, \mathbf{1}, 1,2,3,5,8,0,8,8,3,11,1,12,0,12,12$, $11,10,8,5,0,5,5,10,2,1,3,4,7,11,5,3,8,11$, $6,4,10,1,11,5,3,8,1 \overline{1,6,4,10}, 1,11,12,10$, $9,6,2,8,10,5,2,7,9,3,12,2,1,3,4,7, \ldots$

Lenght of period is 28 with 25 elements before the period, all numbers of $G F(13)$ occur!

$p=17: \mathbf{0}, \mathbf{1}, 1,2,3,5,8,13,4,0,4,4,8,12,3,15,1,16$, $0,16,16,15,14,12,9,4,13,0,13,13,9,5,14$, $2,16,1, \mathbf{0}, \mathbf{1}, 1, \ldots$

Lenght of period is 36 . There are three numbers of $G F(17)$ that do not occur: 7,10,11.

Trying to apply this method (16) also to a circle by taking $L=360^{\circ}$ and e.g. $10^{\circ}$ as unit, one could consider Fibonacci numbers modulo 36 , too:

mod36: 0, 1, 1, 2, 3, 5, 8, 13, 21, 34, 19, 17, 0, 17, 17, $34,15,13,28,5,33,2,35,1, \mathbf{0}, \mathbf{1}, 1, \ldots$

Lenght of period is 23 , only 15 numbers occur, 21 numbers are "gaps".

Remark 10 As the quotient $F_{n+1}^{p} / F_{n}^{p} \in\{0,1, \ldots, p-1\}$ is periodical, the limit does not make sense.

One could also consider the way (i) of subchapter (a) and calculate Fibonacci numbers modulo $\pi$ :

$\bmod \pi: \mathbf{0}, 1,1,2,3,1.8584 \ldots, 1.7168 \ldots, 0.4336 \ldots$, $2.1502 \ldots, 2.5840 \ldots, 1.5929 \ldots, \ldots$

As $\pi$ is transcendent and irrational, this sequence is not periodic.
Conclusion: The presented examples of Galois fields $G F(p)$ show that Fibonacci sequences are periodic independent of the pair of start values. As the set of Fibonacci numbers is countable, Fibonacci numbers modulo $\pi$ must generate "gaps" in the interval $[0, \pi) \subset \mathbb{R}$. Here the questions arise, whether there occurs an attractor or not, and, whether there occur finite intervals as gaps or not.

\section{Fibonacci sequences consisting of complex numbers}

In Sec. 2. we mentioned that one can generalize the concept Fibonacci sequences by $a_{n}=F_{n} a_{1}+F_{n-1} a_{0}$ with $a_{0}, a_{1}$ as general start values. Let us now take $a_{0}:=z_{0}$, $a_{1}:=z_{1} \in \mathbb{C}$ and consider e.g. $z_{0}:=1, z_{1}:=i$ as the pair of start values. We visualize the sequence $\left\{z_{n}\right\}$ in the Gauss plane (which is endowed with a Cartesian frame), see Fig. 4: The Fibonacci numbers $z_{n}=F_{n}+i F_{n+1}$ with odd index $n$ are points of a branch of an equilateral hyperbola $c$, while the numbers with even index $n$ belong to a branch of the conjugate hyperbola $c^{\prime}$. Those hyperbolas have equations

$y^{2}-x y-x^{2}=\mp 1$.

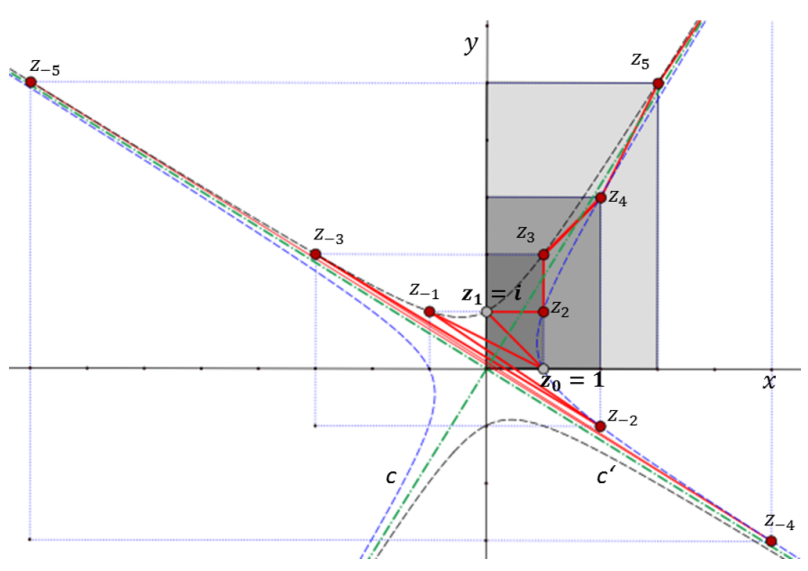

Figure 4: The complex Fibonacci sequence to start values $z_{0}=(1,0), z_{1}=(0,1)$.

The stereographic projection ${ }^{\sigma}$ of these hyperbolas (17) onto the Riemann sphere $\Sigma$ obviously gives a pair of Viviani curves $c^{\sigma}, c^{\prime \sigma} \subset \Sigma$ with common tangents in their common double point $U$, which also acts as the centre of the stereographic projection. Thereby, the stereographic images of the "Fibonacci points" $z_{n}$ of the Gauss plane belong to one loop of each of the two Viviani curves.

Calculating $z=\lim z_{n+1} / z_{n}$ leads to the complex quadratic equation $z^{2}-z-1=0$ with real coefficients. Its solutions 


$$
\begin{aligned}
& s_{1}=a_{1}+i b_{1}, s_{2}=a_{2}+i b_{2} \text { fulfil } \\
& s_{1} s_{2}=1+0 . i, \quad s_{1}+s_{2}=1+0 . i \\
& \Longrightarrow \quad s_{2}=1 / s_{1}=\frac{a_{1}-i b_{1}}{a_{1}^{2}+b_{1}^{2}} \\
& \Longrightarrow \quad a_{1}+i b_{1}+\frac{a_{1}-i b_{1}}{a_{1}^{2}+b_{1}^{2}}=1+0 . i \\
& \Longrightarrow \quad b_{1,2}=0, \quad a_{1,2}=\frac{1}{2}(1 \pm \sqrt{5}) \\
& \Longrightarrow \quad s_{1}=\phi+0 . i, \quad s_{2}=-\phi^{-1}+0 . i .
\end{aligned}
$$

Result 2: Independent of the complex start elements, the $\operatorname{limit} z=\lim z_{n+1} / z_{n}$ of the quotient returns at the (real) Golden Mean $\phi$.

Remark 11 From arbitrary (different) start values $z_{0}, z_{1}$ we get $z_{n}=F_{n} z_{1}+F_{n-1} z_{0},(n \in \mathbb{Z})$. We may speak of $z_{n}$ being a "Fibonacci combination" of $z_{0}, z_{1}$. Consequently, it follows that the Fibonacci points $z_{n}$ belong to a straight line, if $z_{1}=z_{0} \cdot r_{1}, r_{1} \in \mathbb{R}$. In this case the stereographic image on the Riemann sphere $\Sigma$ is circular.

Remark 12 We might relate $\mathbb{C}$ to the two-dimensional vector space $\mathbb{R}^{2}$, as indicated by its visualisation in the Gauss plane. Then the expression $z=\lim z_{n+1} / z_{n}$ must be replaced by $\|\vec{z}\|=\lim \left\|\overrightarrow{z_{n+1}}\right\| /\left\|\overrightarrow{z_{n}}\right\|$. A short calculation shows that this limit again takes the value $\phi \in \mathbb{R}$.

Remark 13 Replacing $\mathbb{C}$ by the ring $\mathrm{D}$ of dual numbers $d:=a+\varepsilon b,\left(\varepsilon^{2}=0, a, b \in \mathbb{R}\right)$ or the ring $\mathrm{A}$ of double numbers $h:=a+j b,\left(j^{2}=1, a, b \in \mathbb{R}\right)$ does not change the visualisation of Fibonacci numbers in the respective Gauss plane. Figure 4 can be considered as a visualisation of dual or double Fibonacci numbers as well. As far as a Fibonacci number is not a zero divisor in $\mathrm{D}$ resp. A, the quotients $d_{n+1} / d_{n}$ resp. $h_{n+1} / h_{n}$ make sense and their limits for $n \rightarrow \infty$ are again $\phi \in \mathbb{R}$. We show this for the case of Fibonacci dual numbers $d_{n+1}=F_{n} d_{1}+F_{n-1} d_{0}$ :

$$
\begin{aligned}
d= & \lim _{n \rightarrow \infty} \frac{d_{n+1}}{d_{n}}=\lim _{n \rightarrow \infty} \frac{F_{n} d_{1}+F_{n-1} d_{0}}{F_{n-1} d_{1}+F_{n-2} d_{0}}=\phi \cdot \frac{\phi d_{1}+d_{0}}{\phi d_{1}+d_{0}} \\
& = \begin{cases}\phi & \text { for } \phi d_{1}+d_{0} \notin N=\{0+\varepsilon b, b \in \mathbb{R}\} \\
- & \text { for } \phi\left(a_{1}+\varepsilon b_{1}\right)+a_{0}+\varepsilon b_{0} \in N\end{cases}
\end{aligned}
$$

From the last equation follows that the condition for a not declared limit reads as

$$
\phi a_{1}+a_{0} \in N, \quad b_{1}, b_{0} \in \mathbb{R} \quad \text { arbitrary. }
$$

The stereographic images of the pair of conjugate equilateral hyperbolas (17) passing through the Fibonacci dual numbers are points of curves of degree 4 on a quadratic cylinder, while Fibonacci double numbers belong to curves on a one-sheeted hyperboloid, c.f. [2].

\section{Fibonacci type sequences defined as vector combinations}

Remark 12 in gives a hint how to define Fibonacci type sequences of vectors:

Recursive definition: Given a set of initial vectors $\left\{v_{0}, v_{1}, \ldots, v_{p}\right\} \subset \mathbb{R}^{k}, p, k \in \mathbb{N}$ and a set of scalars $\left\{r_{0}, \ldots, r_{p}\right\}$, then

$v_{p+r+1}:=r_{0} v_{0+q}+r_{1} v_{1+q}+\cdots+r_{p} v_{p+q}, \quad q \in \mathbb{N}_{0}$.

The sequence obviously can be extended to negative values of $q \in \mathbb{Z}$.

A Fibonnacci type combination of two independent vectors gives "Fibonacci vectors" of a two-dimensional vector space. The Padovan combinations (6) and (8) (i.e., in (19) we put $\left.r_{0}=1, r_{1}=1, r_{2}=0\right)$ applied to three independent start vectors delivers "Padovan vectors" of a 3 -space, which are recursively defined by

$v_{n}=v_{n-1}+v_{n-2}=P_{n-2} v_{0}+P_{n} v_{1}+P_{n-1} v_{2}$.

We present an example using the basis vectors of $\mathbb{R}^{3}$ as the start vector triplet, i.e

$v_{0}=(1,0,0)^{\top}, \quad v_{1}=(0,1,0)^{\top}, \quad v_{2}=(0,0,1)^{\top}$,

such that (20) becomes

$v_{n+1}=\left(P_{n-3}, P_{n-1}, P_{n-2}\right)^{\top}$.

For the following visualisations in Figure 5, it seems to be necessary to calculate the Padovan numbers at least for $-40 \leq n \leq 19$, see Table 1 :

Table 1: List of Padovan numbers for $-40 \leq n \leq 19$

\begin{tabular}{|c|ccccccccc|}
\hline$-40 \ldots-31$ & 145 & -89 & 56 & -7 & -33 & 49 & -40 & 16 & 9 \\
$-30 \ldots-21$ & -24 & 25 & -15 & 1 & 10 & -14 & 11 & -4 & -3 \\
$-20 \ldots-11$ & 7 & -7 & 4 & 0 & 4 & -3 & 1 & 1 & -2 \\
$-10 \ldots-1$ & 2 & -1 & 0 & 1 & -1 & 1 & 0 & 0 & 1 \\
$0 \ldots 9$ & $\mathbf{0}$ & $\mathbf{1}$ & $\mathbf{1}$ & 1 & 2 & 2 & 3 & 4 & 5 \\
$10 \ldots 19$ & 7 & 9 & 12 & 16 & 21 & 28 & 37 & 49 & 65 \\
\hline
\end{tabular}

Remark 14 The Padovan numbers resp. vectors with negative index $n$ are recursively defined by

$Q_{m+1}=Q_{m-2}-Q_{m}, \quad(m=-n)$ resp. $v_{m+1}=v_{m-2}-v_{m}$.

Thereby, it follows that $\lim _{m \rightarrow \infty} \frac{Q_{m-1}}{Q_{m}}=\frac{1}{\psi}$ is a solution of $y^{3}+y^{2}-1=0$. 
Remark 15 The (real) vector $\left(1, \psi^{2}, \psi\right)^{\top}, \psi=1,32 \ldots$ the van der Laan number, points to $v_{\infty}$. Equation (7) for van der Laan's Plastic Number $x_{1}=\psi$ has two complex conjugate solutions of the quadratic equation

$x^{2}+\psi x+\left(\psi^{2}-1\right)=0$,

$x_{2,3}=\frac{1}{2} \cdot\left(-\psi \pm \sqrt{4-3 \psi^{2}}\right) \approx-0,662359 \pm i \cdot 1,12456$.

The correspoding complex vectors $\left(1, x_{2,3}^{2}, x_{2,3}\right)^{\top}$ represent complex ideal points of the spiral set of points described by (21) for $m \rightarrow \infty$, see Fig. 5 visualising the top view of that point set. The star-shaped blue polygon tends to a pentagram for $m \rightarrow \infty$. (The black, almost straight lined polygon describes the top view of the first $n$ points, $n>2$.) One might calculate the angle $\alpha:=\angle\left(v_{n-1}-v_{n}\right)\left(v_{n+1}-v_{n}\right)$ of the limit 3D-pentagram's spikes. It turns out that one gets

$\cos \alpha=\frac{\psi(2 \psi-1)}{\psi^{2}+\psi+1} \quad \Longrightarrow \quad \alpha \approx 68,0 \ldots \circ$

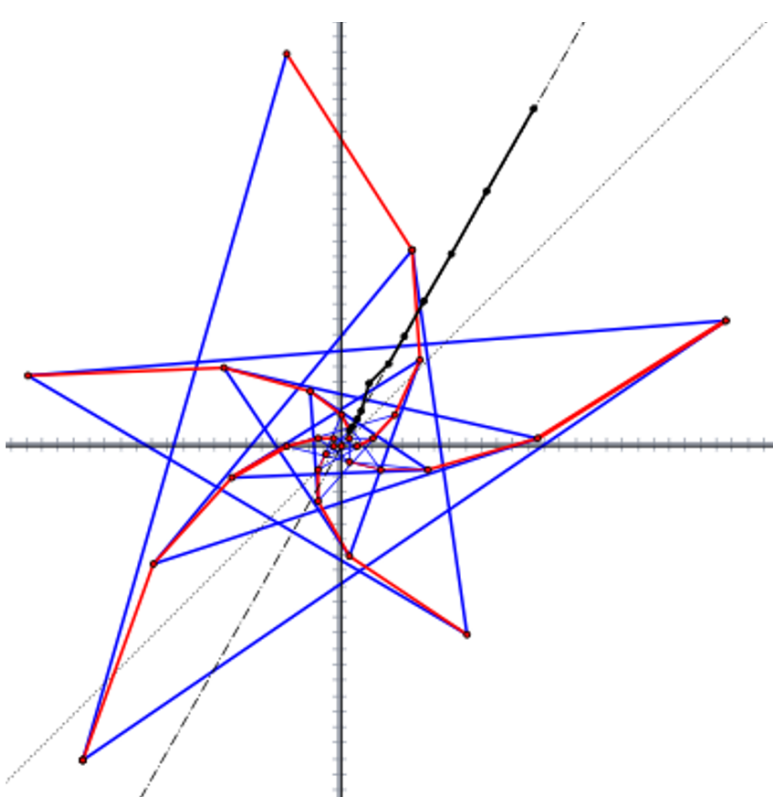

Figure 5: Top view of the Padovan type point set resulting from (20).

Remark 16 Obviously one can interpret the set of vectors $v_{n}$ (19) as homogeneous coordinates of points of a projective space $\pi=\mathrm{P}\left(\mathbb{R}^{k}\right)$. Again we focus on the example of Padovan vectors as treated above. We replace the vector symbol $v_{n}$ by the symbol $V_{n}$ for projective points and continue the "Padovan example" above rewriting (21) as

$V_{n+1}=\left(P_{n-3}, P_{n-1}, P_{n-2}\right) \mathbb{R}$.

\section{Normed Fibonacci combinations and Con- clusion}

The interpretation in Remark 16 stimulates to consider Fibonacci or Padovan type combinations of Matrices or even (algebraic) equations too. The Fibonacci combination of e.g. two such geometric objects $O b j_{0}, O b j_{1}$ of the same type results in a set of objects belonging to a pencil

$O b j_{n}=F_{n} O b j_{1}+F_{n-1} O b j_{0}$

Similarly, the Padovan-combination of three such objects results in a set of these objects belonging to a twoparameter manifold. As one can interpret the coefficients $F_{n}, F_{n-1}$ in (26) as projective coordinates within the pencil of objects, the limit object $O b j_{\infty}$ for $n \rightarrow \infty$ also makes sence. As an example we show this situation for the Fibonacci combination of two circles $c_{0}, c_{1}$ :

$c_{0} \ldots\left(x-m_{0}\right)^{2}+y^{2}=r_{0}^{2}, \quad c_{1} \ldots\left(x-m_{1}\right)^{2}+y^{2}=r_{1}^{2}$,

$\left(\begin{array}{ccc}m_{0}^{2}-r_{0}^{2} & -m_{0} & 0 \\ -m_{0} & 1 & 0 \\ 0 & 0 & 1\end{array}\right), \quad\left(\begin{array}{ccc}m_{1}^{2}-r_{1}^{2} & -m_{1} & 0 \\ -m_{1} & 1 & 0 \\ 0 & 0 & 1\end{array}\right)$.

To receive the normed equation for the circle $c_{n}$ we have to "norm" the Fibonacci combination as in (26):

$c_{n}=\frac{F_{n-1}}{F_{n-1}+F_{n}} c_{0}+\frac{F_{n}}{F_{n-1}+F_{n}} c_{1}$,

and we get

$c_{\infty}=\frac{1}{\phi^{2}} c_{0}+\frac{1}{\phi} c_{1}$

Using power rules for $\phi$, we receive the normed equation for that limit circle $c_{\infty}$

$c_{\infty} \ldots\left(x-\left(\frac{m_{0}}{\phi^{2}}+\frac{m_{1}}{\phi}\right)\right)^{2}+y^{2}=\frac{r_{0}^{2}}{\phi^{2}}+\frac{r_{1}^{2}}{\phi}-\frac{1}{\phi^{3}}\left(m_{0}-m_{1}\right)^{2}$.

From (29), we read off that the center $M_{\infty}$ of $c_{\infty}$ divides the segment between the centres $M_{0}, M_{1}$ of $c_{0}, c_{1}$ in the Golden Ratio. Note that the radius $r_{\infty}$ and thus $c_{\infty}$ can be complex, if $c_{0}, c_{1}$ span a hyperbolic pencil of circles! In case $c_{\infty}$ is real, it must intersect the circles of the elliptic pencil of circles conjugate to that spanned by $c_{0}, c_{1}$ orthogonally. In case $c_{0}, c_{1}$ span an elliptic or parabolic pencil of circles, $c_{\infty}$ has to pass throught the basis point(s) of that pencil. So it is easy to construct $c_{\infty}$ to a given pair $c_{0}, c_{1}$, see Fig. 6 .

In the following, we focus on the norming process used in (27) and we will speak of "normed Fibonacci combinations" of two (geometric) objects. 

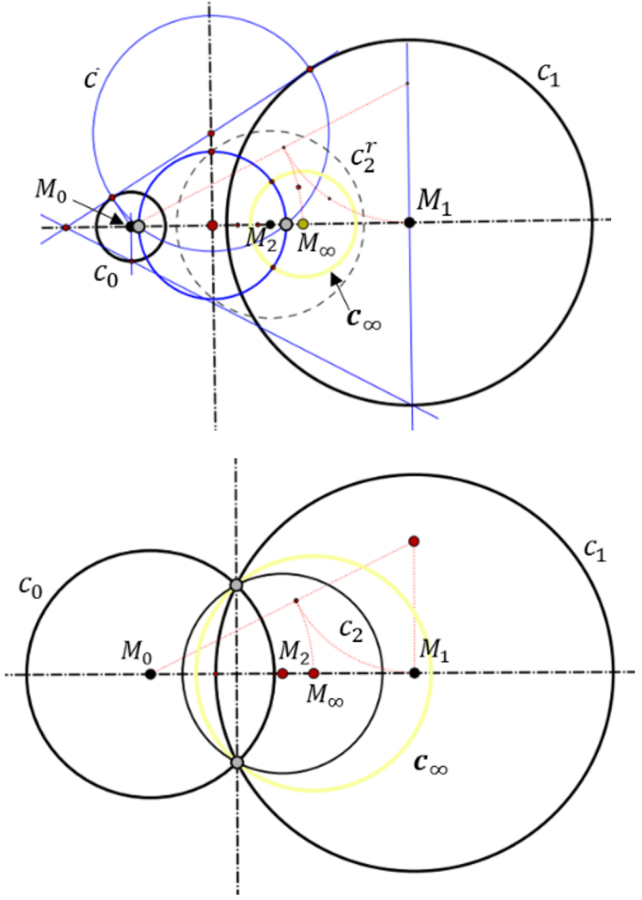

Figure 6: Limit circle $c_{\infty}$ to The Fibonacci combination of two circles $c_{0}, c_{1}$.

top: $c_{0}, c_{1}$ span a hyperbolic pencil of circles, $\bar{c}$ is a circle of the (elliptic) conjugate pencil, $c_{\infty}$ is real, but $c_{2}$ is imaginary with the "real representative" $c_{2}^{r}$.

bottom: $c_{0}, c_{1}$ span an elliptic pencil of circles. All circles $c_{n}$ are real.

a) Let the given objects $O b j_{0}, O b j_{1}$ be $(n \times m)$ matrices. For $n=m$, they can be interpreted as the coordinate representation of collineations, while for $n>m$ they represent linear mappings. A Fibonacci combination (26) of such matrices represents a collineation resp. a linear mapping which depends on the ratio $F_{n}: F_{n-1}$ and the two initial matrices $O b j_{i}$. Thus, the resulting mapping also has the "normed Fibonacci representation"

$O b j_{n}=\frac{F_{n-1}}{F_{n+1}} O b j_{0}+\frac{F_{n}}{F_{n+1}} O b j_{1}$

and we call $O b j_{n}$ the " $n^{\text {th }}$ Fibonacci mean" of $O b j_{0}$ and $O b j_{1}$. For the object $O b j_{\infty}$ the name "Golden Mean of $O b j_{0}$ and $O b j_{1}$ " could be coined.

b) Let the given objects $O b j_{0}, O b j_{1}$ be vectors, the coordinates of which fulfil a (quadratic) condition, then the coordinates of the Fibonacci or Padovan type combination $O b j_{n}$ will not fulfil this condition anymore! Examples of such vectors are, e.g., the Plücker coordinates of lines of a projective 3-space, the spear- and cycle- resp. circle-coordinates in Laguerre, Möbius, and Liegeometry (see [2]). Here a "norming" of the resulting vector $O b j_{n}$ analogue to that used in (25) would allow to extend the concept "pencil" or "two-parameter manifold" as well as "Fibonacci mean" and "Padovan mean" also to these cases.

For example, in the case of Line Geometry the Fibonacci combination of two skew lines $g_{0}, g_{1}$ results in a set of linear complexes within the pencil of linear complexes spanned by $g_{0}, g_{1}$. Here the norming process could consider the axes $a_{n}$ of these complexes $O b j_{n}$, if the space of action is the projective closure of the Euclidean 3-space. As the set of axes of a pencil of linear complexes comprises the rulings of a Plücker conoid, the axis $a_{\infty}$ of the "limit complex" $O b j_{\infty}$ is a line "in Golden Ratio" between $g_{0}$ and $g_{1}$. We might call $a_{\infty}$ the Golden Mean line of the lines $g_{0}$ and $g_{1}$.

c) Coming back to numbers we put $O b j_{i}:=\log q_{i}$. Now (30) becomes

$$
\begin{gathered}
\log q_{n}=\frac{F_{n-1}}{F_{n+1}} \log q_{0}+\frac{F_{n}}{F_{n+1}} \log q_{1}, \\
n \in \mathbb{N}, \quad q_{0}, q_{1} \in \mathbb{R}^{+},
\end{gathered}
$$

what means that

$$
q_{n}=\sqrt[F_{n+1}]{q_{0}^{F_{n-1}} \cdot q_{1}^{F_{n}}}
$$

and finally

$q_{\infty}=q_{0}^{1 / \phi^{2}} \cdot q_{1}^{1 / \phi}$

Hereby, we receive as a final

Result 3: The geometric mean of two (ordered) numbers $q_{0}, q_{1}$ can be interpreted as the third normed Fibonacci mean $q_{3}$ of these numbers, while $q_{\infty}$ is their Geometric Golden Mean.

Concluding remark. The presented material does not at all cover all possibilities to generalise the Fibonacci sequence. By focussing on both, the Fibonacci sequence and the Padovan sequence the material connects to known facts which are seen from a perhaps new point of view. Obviously the presented methods can be applied to any Fibonacci (resp. Padovan) type sequence. It might be worth to treat the sequence leading to V. de Spinadel's Silver Mean more explicite and in the above presented way.

\section{Acknowledgement}

The author thanks Ema Jurkin and the reviewer(s) for their valuable support and suggestions. 


\section{References}

[1] V.S. ADAmchoK, D.J. JEFFrey, Polynomial Transformations of Tschirnhaus, Bring and Jerard, ACM SIGSAM Bulletin 37(3) (2003), 90-94.

[2] W. BENZ, Vorlesungen über Algebren, (Grundlehren der math. Wiss., Vol. 197), Springer-Verlag Berlin, 1973.

[3] H.S.M. Coxeter, Problem E3007 (due to G. ODOM), American Math. Monthly 1983.

[4] M. Hazewinke, Lucas polynomials, in Encyclopedia of Mathematics, Springer Science + Buisiness Media B.V. Kluwer Academic Publishers, 2001.

[5] R. Padovan, Dom Hans van der Laan and the Plastic Number, Nexus Network Journal 4(3), Architecture and Mathematics (2002), 181-193.

[6] A. Redondo-Buitrago, Sobre los systemas de proporciones áureo y plastic y sus generalisaciones, Journ. Mathematics \& Design 9(1) (2009), 15-34.

[7] L. Rosenbusch, Räumliche Proportionen, in $G e$ ometry, Art, and Science 06, ed. O. NIEWIADOMSKI, Hauschild Verlag, 2007.

[8] V.W. DE SPINADEL, The Family of Metallic Means, Visual Mathematics 1(3) (1999).

[9] V.W. DE SPINADEL, Golden and Metallic Means in Modern Mathematics and Physics, Proc. 13th ICGG 2008, Dresden, Germany, 2008.

[10] V.W. DE SPINADEL, G. WEISS, Remarks to classical cubic problems and the Mean Values of van der Laan and Rosenbusch, Proc. 14th ICGG, Kyoto, Japan, 2010.

[11] G. WeIss, Ratios and Mean Values: A Topic in Art, Architecture, and Mathematics, Proc. 29th Conf. on Geom. \& Graph., Doubice, Czech Republic, 2009.

[12] G. WEISS, Remarks on Fractions, Fractals, and Golden Sections, Journ. Mathematics \& Design 8(2) (2008).

[13] G. WeIss, S. Mick, Non-standard Visualisations of Fibonacci Numbers and the Golden Mean, KoG 18 (2014), 34-42.

[14] G. Weiss, V.W. DE SPINAdEL, From George Odom to a New System of Metallic Means, Journ. Mathematics \& Design 13 (2014), 71-85.

[15] Wikipedia, https://en.wikipedia.org/wiki/ Fibonacci-number

[16] Wikipedia, https://en.wikipedia.org/wiki/ Padovan_sequence

\section{Gunter Weiss}

e-mail: weissgunter@hotmail.com

University of Technology Vienna,

Karlsplatz 13, 1040 Vienna, Austria

University of Technology Dresden,

Helmholtzstrße 10, 01069 Dresden, Germany 